\title{
Outcomes of a Transitional Care Clinic to Reduce Heart Failure Readmissions at an Urban Academic Medical Center
}

Justin Lee, Felix Reyes, Minhazul Islam, Mafuzur Rahman, Miguel Ramirez, Jonathan Francois and Samy I. McFarlane Department of Medicine, SUNY Downstate Health Science University, Brooklyn, NY 11203, USA

\begin{abstract}
Heart Failure (HF) is one of the leading hospital readmission diagnoses in the United States. It is a major challenge in today's healthcare environment to reduce hospital readmissions for HF and much of the expenditure on HF is on in-hospital treatment. In the USA, risk factors for readmission with HF include being African American, low-socioeconomic status, Medicare, Medicaid, self-pay/no insurance and drug abuse. The Transitional Care Clinic (TCC) model established at our institution integrated multiple facets of chronic HF management, including early post-discharge follow-up, phone call reminders as well as clinical pharmacists and nurse practitioner's integration into the treatment team.
\end{abstract}

Of $488 \mathrm{HF}$ admissions to our institution from March 2015 until May 2017, mean age $=65$ years (SD 13.03), 262 patients were males (53.6\%) and 463 patients (94\%) were Blacks. There was a total of 121 readmissions within 30 days after discharge $(24.8 \%)$ and 43 readmissions 7 days after discharge $(8.81 \%)$ during our study period. 159 patients (32.58\%) followed up in our TCC, while 329 patients $(67.41 \%)$ did not at TCC. Within 7 days post discharge, there was 3 (1.9\%) Vs $40(12.2 \%)$ readmissions for TCC and non-TCC groups respectively, $\mathrm{P}<0.01$. There was $18(11.32 \%)$ Vs $103(31.31 \%)$ readmissions within 30 days post discharge for TCC and non-TCC groups respectively $\mathrm{P}<0.01$.

Among high readmission risk and predominantly black population with HF, TCC resulted in significantly lower hospital readmission rate within 7 days and within 30 days of initial discharge. These data help inform policy makers regarding the effectiveness of TCC model for resource allocation and broader implementation, particularly among high risk population with the potential of cost saving and better patient outcomes.

\section{Introduction}

In the United States, Heart Failure (HF) is one of the leading chronic illnesses with a prevalence of 5 million patients [1]. The cost of healthcare expenditure on heart failure treatment is astronomical, with an estimated cost of $\$ 31$ billion in 2012 and a projected increase of up to $\$ 69.7$ billion by 2030 [2]. A vast majority of this expenditure is towards hospital admissions, as complications leading to decompensated HF, including acute respiratory distress, multi-organ systemic dysfunction and adverse events from polypharmacy are often considered too complicated and deemed high-risk for outpatient care. One of the challenges for health care providers and policy makers is how to reduce hospitalization rate for HF, particularly with our aging populations, the hospitalization rate for HF treatment would only be expected to rise since HF increases with age [3].

In the US, much of the aforementioned expenditure in HF treatment is spent in hospital readmissions, as approximately $25 \%$ of initial HF admissions will be followed by a second admission [1]. These challenges led to the initiation of the Hospital Readmissions Reduction Program (HRRP) of the Patient Protection and Affordable Care Act. Beginning in 2012, institutions with unacceptably high 30day readmission rates for several conditions, including HF, were faced with financial penalties under this legislation [4].

Previous studies have also shown that black population with low socioeconomic status has significantly higher incidence rate of HF than other ethnicities in the US [3]. Not only is there a racial discrepancy in the proportional number of HF patients, but there is also a striking difference in the rate of hospitalization amongst different racial groups in HF patients. A recent TOPCAT (Treatment of Preserved Cardiac Function Heart Failure With an Aldosterone

\section{Publication History:}

Received: December 01, 2019

Accepted: December 28, 2019

Published: December 30, 2019

\section{Keywords:}

Heart failure, Transition of care, Readmission
Antagonist) trial from American Heart Association has shown that self-reported black patients have higher risk for hospitalization than non-blacks (HR: 1.51, CI: 1.167-1.97; $P=0.002$ ) [5].

It is interesting to note that a previous large-scale cohort study analyzing characteristics of HF patients in the US showed that African American race, low-socioeconomic status, Medicare, Medicaid, selfpay/no insurance and drug abuse were all associated with higher readmission risk after hospitalization for CHF [6].

Our central Brooklyn community consists of $80 \%$ black ethnicity, with $31 \%$ living below poverty level. Sixty percent of residents of this community aged 25 or older do not have a college degree. Managing patients with chronic medical conditions in our community with low health literacy is particularly challenging, as many of them do not fully understand the complexity of their illness and the importance of adherence with medical treatment plan. Reasons for hospital admissions for HF exacerbation include not only medication non-compliance, but also failure to follow up with primary care appointments and inappropriate use of the emergency room as the medium for immediate care [7].

"Corresponding Author: Prof. Samy I. McFarlane, Division of Endocrinology, Department of Internal Medicine, State University of New York, Downstate Medical Center, Brooklyn, New York,11203, USA, Tel: 718-270-6707, Fax: 718270-4488; E-mail: smcfarlane@downstate.edu

Citation: Lee J, Reyes F, Islam M, Rahman M, Ramirez M, et al. (2019) Outcomes of a Transitional Care Clinic to Reduce Heart Failure Readmissions at an Urban Academic Medical Center. Int J Clin Res Trials 4: 140. doi: https://doi. org/10.15344/2456-8007/2019/140

Copyright: (C) 2019 Lee et al. This is an open-access article distributed under the terms of the Creative Commons Attribution License, which permits unrestricted use, distribution, and reproduction in any medium, provided the original author and source are credited. 
Citation: Lee J, Reyes F, Islam M, Rahman M, Ramirez M, et al. (2019) Outcomes of a Transitional Care Clinic to Reduce Heart Failure Readmissions at an Urban Academic Medical Center. Int J Clin Res Trials 4: 140. doi: https://doi.org/10.15344/2456-8007/2019/140

Page 2 of 5

Our institution has established a transitional care clinic (TCC) in May 2015, with aims to aid patients with chronic medical conditions by providing early post-discharge follow up regardless of patient's financial or insurance status.

Previous transitional care models have proven success in reducing all-cause intermediate-term and long-term readmissions for chronic illnesses, not just HF [8].

Other studies have shown home-visiting programs and structured telephone support interventions reduced HF-specific readmission with a mortality benefit [9]. Not only does it decrease the number of readmissions, but it also has been shown that transitional care programs for HF patients can improve the individual's quality of life and decrease the overall cost of care [10].

With this in mind, our TCC model was designed to integrate multiple facets of transitional care program that have proven to be beneficial for HF patients, including early post-discharge follow-up $(<$ 7 days), phone call reminders a day prior to follow-up appointment, post-clinic follow up phone call, establishment of clinical pharmacists to provide medication reconciliation and nurse practitioners to address symptoms, monitor vital signs, schedule additional appointments and to educate patients on the importance of medication compliance and lifestyle modifications.

\section{Materials and Methods}

\section{Setting}

State University of New York (SUNY) Downstate Health Science University is an 882-bed teaching hospital located in Brooklyn,
New York. Admitted patients to general Internal Medicine floors are distributed throughout 6 medical-surgical nursing stations. General Internal Medicine patients are admitted directly through the Emergency Department and are assigned to a team lead by an ABIM Board Certified Hospitalist. Upon discharge, patients can follow in one of 75 outpatient clinics depending on their medical needs. All study subjects had a previous diagnosis of CHF.

\section{Interventions}

Prior to discharge, patients with a primary diagnosis of CHF exacerbation were identified by the Internal Medicine team. Upon discharge, patients were provided with a follow up appointment with TCC within 7 days from the discharge date. All patients received follow up reminder through a phone call 1 day prior to their TCC appointment. Our TCC clinic was open Mondays and Fridays of every week, excluding holidays. At the TCC, the patient interacts with 3 health care providers: a clinical pharmacist, a nurse and a physician. The clinical pharmacist reviews the patient's medications and ensures accurate understanding of schedules and use. Nurse practitioner addresses symptoms, monitors vital signs and schedules additional appointments if necessary. Lastly the physician performs a physical examination and address old or new health symptoms/ issues in the care of the patient.

\section{Study of the intervention}

Readmissions rates between HF patients who followed up at their TCC appointments were compared to non-TCC control. Our Study period was from March 2015 until May 2017.

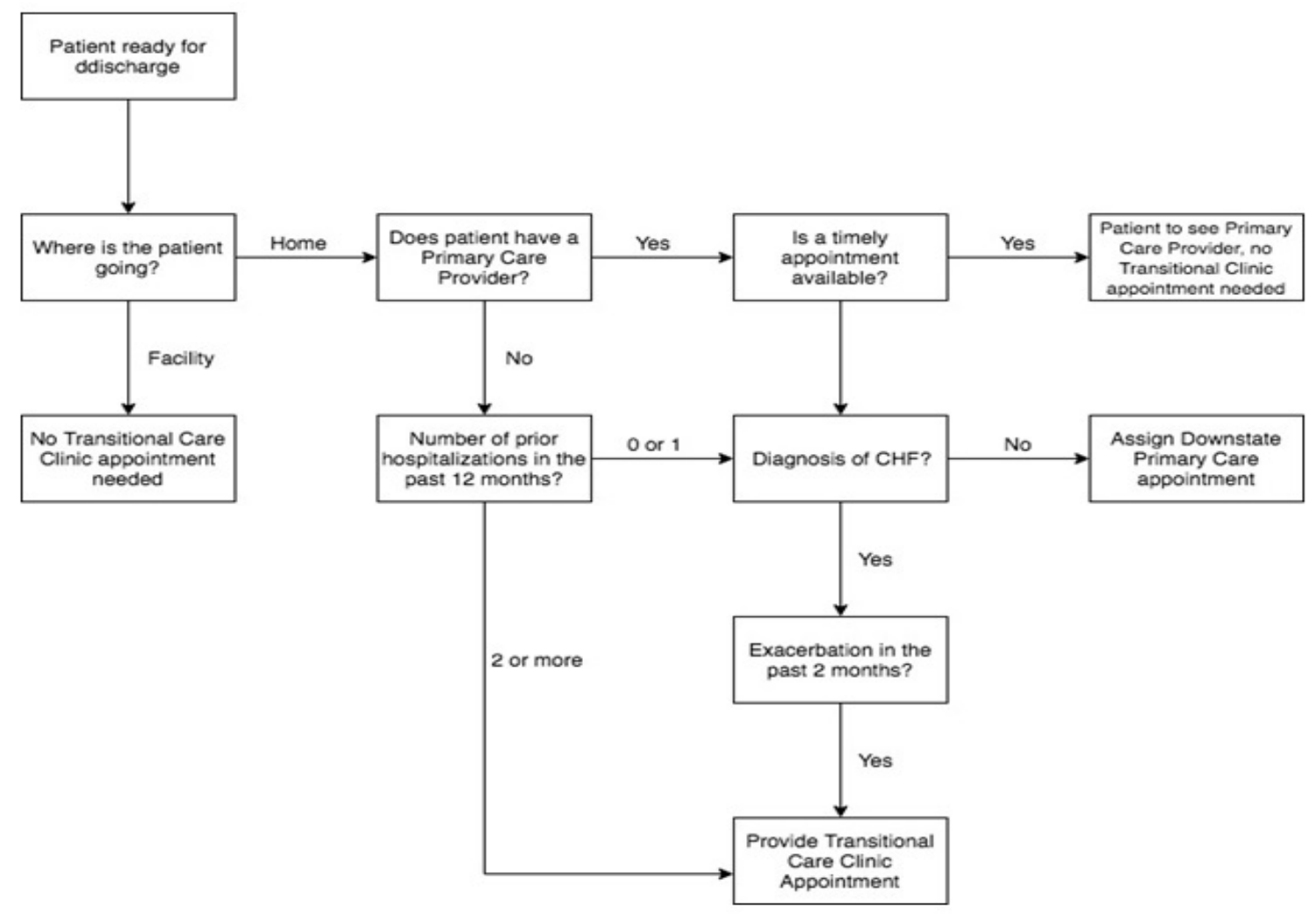

Figure 1: Transitional Care Clinic referral algorithm. 
Citation: Lee J, Reyes F, Islam M, Rahman M, Ramirez M, et al. (2019) Outcomes of a Transitional Care Clinic to Reduce Heart Failure Readmissions at an Urban Academic Medical Center. Int J Clin Res Trials 4: 140. doi: https://doi.org/10.15344/2456-8007/2019/140

Page 3 of 5

\section{Measures}

Three investigators (FR, JL and $\mathrm{MI}$ ) were trained to abstract data from the electronic medical record. A standardized data abstraction form was used which included: patient demographics, dates of hospital admission, discharge, readmission, ED visits, length of hospitalization, and comorbidities. 30-day readmission rate was calculated using this information. The 30 -day readmission rate was chosen as an outcome measure based on CMS guidelines.

\section{Analysis}

We analyzed the data using IBM SPSS Statistics version 23 (IBM (C). Categorical values were reported as counts and percentages and continuous variables were reported as means and SDs, or as medians with interquartile range, as appropriate. For comparison, $\chi^{2}$ tests were used to determine statistical significance among groups, which was set at $\mathrm{P}<0.05 .30$-day readmission rate was calculated and compared between patients who followed up at their TCC and who did not by Chi-squared and Fisher's exact test. Also, readmission rates were compared for patients who followed at TCC vs. cardiology clinic follow up, as well as for those who had cardiology service consult as inpatient vs. those who did not receive consultations.

\section{Ethical Considerations}

The intervention posed no theoretical harm to patients. The burden of implementing this model falls upon new providers that were trained in the TCC workflow. There was no invasion of privacy to patients or providers beyond what is expected in a regular hospital admission. No additional clinical burden was placed upon medical providers (residents or hospitalists), as obtaining information regarding primary care providers and follow up is standard procedure in daily practice. The study protocol was approved by the ethics committee (SUNY Downstate Medical Center - Institutional Review Board).

\section{Results}

Our sample size consisted of $488 \mathrm{HF}$ admissions to University Hospital of Brooklyn, Downstate Health Science University. There was a total of 121 readmissions 30 days after discharge (24.8\%) and 43 readmissions 7 days after discharge (8.81\%) during our study period.

\section{Demographics}

The average age of our study was 65 years (SD 13.03). 262 patients were male (53.6\%) and 226 were female (46.3\%). 463 patients (94\%) were African American.

159 patients (32.58\%) followed up in our transitional care clinic, while 329 patients $(67.41 \%)$ did not show to their post-discharge appointment. Of those who showed up at their appointment only 18 were readmitted $(11.32 \%)$. Of those who didn't show up at their appointment 103 were readmitted (31.31\%). A chi-square test of independence was performed which revealed a significant relation between following at TCC and no readmissions within 30 days postdischarge, $\mathrm{X} 2(1, \mathrm{~N}=488)=22.967, \mathrm{p}<0.05$.

\section{Discussion}

Many reasons have been cited as causes of high readmission rates: inefficient transitions of care, lack of availability in post-discharge follow up, inadequate access to medications, therapy non-adherence and dietary indiscretions [9].

Disease management programs have been implemented with different modalities and different degrees of success. The different modalities of outpatient disease management for patients with CHF include: at home interventions, outpatient visits, telephone support, non-invasive telemonitoring and invasive telemonitoring [11]. In populations with limited literacy and access to telecommunications such as the low-income residents of Brooklyn, outpatient visits

\begin{tabular}{|c|c|c|c|c|}
\hline Characteristic & All Cohort & TCC Follow up & TCC No Show & $\mathrm{P}$-value \\
\hline \multicolumn{5}{|l|}{ Patients } \\
\hline Total number & 488 & 159 & 329 & \\
\hline Age in years & 65.0 & 65.9 & 64.5 & $0.23^{\mathrm{a}}$ \\
\hline$<35$ & 15 & 6 & 9 & \\
\hline $35-50$ & 46 & 6 & 40 & \\
\hline $51-65$ & 186 & 67 & 119 & \\
\hline $66-80$ & 192 & 63 & 129 & \\
\hline$>80$ & 49 & 17 & 32 & \\
\hline Sex & & & & $0.902^{\mathrm{b}}$ \\
\hline Males & 262 & 86 & 176 & \\
\hline Females & 226 & 73 & 153 & \\
\hline Race & & & & $0.207^{\mathrm{b}}$ \\
\hline African American & 463 & 154 & 309 & \\
\hline Hispanic & 12 & 0 & 9 & \\
\hline White & 9 & 4 & 8 & \\
\hline Asian & 4 & 1 & 3 & \\
\hline
\end{tabular}


Citation: Lee J, Reyes F, Islam M, Rahman M, Ramirez M, et al. (2019) Outcomes of a Transitional Care Clinic to Reduce Heart Failure Readmissions at an Urban Academic Medical Center. Int J Clin Res Trials 4: 140. doi: https://doi.org/10.15344/2456-8007/2019/140

Page 4 of 5

\begin{tabular}{|c|c|c|c|c|c|c|c|}
\hline & Total & $\begin{array}{l}\text { 7-day } \\
\text { Readmission }\end{array}$ & $\begin{array}{l}\chi^{2-\text { statistic }^{a}} \\
\text { (df) }\end{array}$ & P-value & $\begin{array}{l}\text { 30-day } \\
\text { readmission }\end{array}$ & $\begin{array}{l}\chi^{2-\text { statistic }^{a}} \\
\text { (df) }\end{array}$ & P-value \\
\hline $\begin{array}{l}\text { Transitional care } \\
\text { follow up }\end{array}$ & 159 & $3(1.89 \%)$ & 15.537 & $<0.001$ & $18(11.32 \%)$ & 22.967 & $<0.001$ \\
\hline $\begin{array}{l}\text { No Transitional } \\
\text { care follow up }\end{array}$ & 329 & $40(12.16 \%)$ & & & $103(31.31 \%)$ & & \\
\hline $\begin{array}{l}\text { Cardiology } \\
\text { consult }\end{array}$ & 130 & $11(8.46 \%)$ & 3.85 & 0.427 & $36(27.69 \%)$ & 1.403 & 0.844 \\
\hline $\begin{array}{l}\text { No Cardiology } \\
\text { consult }\end{array}$ & 349 & $30(8.6 \%)$ & & & $83(23.78 \%)$ & & \\
\hline $\begin{array}{l}\text { Cardiology } \\
\text { follow up }\end{array}$ & 102 & $2(1.96 \%)$ & 13.788 & 0.008 & $8(7.84 \%)$ & 31.841 & $<0.001$ \\
\hline $\begin{array}{l}\text { No Cardiology } \\
\text { follow up }\end{array}$ & 189 & $24(12.7 \%)$ & & & $68(35.98 \%)$ & & \\
\hline
\end{tabular}

Table 2: 7-day and 30-day HF readmissions.

${ }^{\text {a }}$ Chi-square test for independence

might be more effective than tele-medicine interventions that would require access to a working telephone or understanding of software use. Unfortunately, no solution is free of its own shortcomings; in our study population, only $35 \%$ of patients presented to their TCC appointment. Several factors unique to our population could explain these findings: our lowest income patients might have to face difficult choices at the time of deciding to follow up with the providers due competing interests.

According to anecdotal reports from our patients, most have to take into consideration the amount of money that they will not earn from missing a day of work if they decide to show up at their appointment. At the same time, they have additional expenses related to the clinic visit such as transportation, insurance co-payments, etc. For residents in this community, a missed day of work could lead to lack of earnings necessary to provide food for their family. Therefore, it was important for us to perform this study and not to adopt models from other communities with different patient populations and available resources.

Outpatient clinic-based care can take many forms. Patients could follow at multidisciplinary HF clinics, nurse-led HF clinic or primary care. In a systematic review it was noted that few studies ever describe the type of outpatient care that HF received [9]. Only one randomized control trial by Oddone et al. has evaluated the effectiveness of outpatient clinic-based care led by a primary care provider in the past [12]. In this trial, enhanced access to primary care providers led to no change in perceived health status by patients or in the rate of readmissions. A possible explanation for the discrepancy between the results of this trial and our findings is the significant difference between the study populations. Oddone et al. study was based in nine veteran affairs (VA) hospitals, where veterans had guaranteed access to healthcare institutions within the VA network, meanwhile our study was performed in a high-risk population with poor access to quality care.

Recently, a clustered randomized controlled trial conducted by Englander et al. investigated such impact of multidisciplinary approach for a targeted population that closely resembles our study population, at a single urban medical center in Portland, Oregon [13]. Their multidisciplinary intervention, named C-TraIn (Care Transitions Innovation), included transitional nurse coaching and education, with provision of medication for up to 30 days after initial discharge regardless of the insurance or financial status of the patient, which was very similar to our approach in the TCC. Interestingly, in this certain population, their particular transitional care model did not significantly reduce 30 -day inpatient readmissions or ED use. However, such multicomponent intervention has shown to improve subjective quality of care transition, reported by the patients of this under-privileged population [13].

The results of our study reflect in part, a combination of evidence presented by all the previous randomized clinical trial outlined on the optimal disease management in CHF patients. Outpatient visits can reduce hospital admissions and be cost-effective, if implemented correctly within disease management programs [11]. Of these studies, only two studies have shown decrease in HF related readmissions, $[14,15]$ while five showed reduction in all-cause readmissions only $[16,14,17,18,15]$.

\section{Limitations}

A limiting factor of our study was the poor clinic follow up in our study population. The patients who follow up at clinic could be more likely to be compliant with other therapeutic interventions and therefore less likely to be admitted.

\section{Conclusion}

From our 2-year investigation of the effectiveness of transitional care clinic model, developed and implemented on heart failure patients within a population almost entirely comprised of socioeconomically under-privileged and racial minority group, we have found that our TCC model significantly reduces the rate of CHF-related readmission within 7 and 30 days of initial discharge. CHF-related readmission rates were not significantly different between those patients who were enrolled in our TCC model, compared to the patients who were followed up by cardiology service, showing non-inferiority of our multidisciplinary service, largely operated by physician assistants and clinical pharmacists. This result suggests that the most important aspect of reducing costly readmissions in this extremely complicated and difficult to manage condition is ensuring timely follow up. Future policies and allocation of health care budget on CHF management should be focused heavily on providing easy access to health care providers or a team comprising professionals from multitudes of discipline, especially in an at-risk population. 
Citation: Lee J, Reyes F, Islam M, Rahman M, Ramirez M, et al. (2019) Outcomes of a Transitional Care Clinic to Reduce Heart Failure Readmissions at an Urban Academic Medical Center. Int J Clin Res Trials 4: 140. doi: https://doi.org/10.15344/2456-8007/2019/140

Page 5 of 5

\section{Future Directions}

Future directions of our transitional clinic project will be targeted towards increasing the show up rate in our population or offering home visits during non-working hours to those patients who cannot come to the clinic.

\section{Funding}

This work is supported, in part, by the efforts of Dr. Moro O. Salifu M.D., M.P.H., M.B.A., M.A.C.P., Professor and Chairman of Medicine through NIH Grant number S21MD012474.

\section{Competing Interests}

The authors declare that they have no competing interests.

\section{References}

1. Roger VL (2013) Epidemiology of Heart Failure. Circ Res 113: 646-659.

2. Heidenreich PA, Albert NM, Allen LA, Bluemke DA, Butler J, et al. (2013) Forecasting the Impact of Heart Failure in the United States. Circ Heart Fail 6: $606-619$

3. Chen J, Normand SL, Wang Y, Krumholz HM (2011) National and regiona trends in heart failure hospitalization and mortality rates for Medicare beneficiaries, 1998-2008. Jama 306: 1669-1678.

4. Smith K, Fleming JP, Gros B (2018) Editorial: Transitional Care Clinics to Reduce 30-day Readmissions in Heart Failure Patients. Cureus 10: e2069.

5. Pitt B, Pfeffer MA, Assmann SF, Boineau R, Anand IS, et al. (2014) Spironolactone for Heart Failure with Preserved Ejection Fraction.

6. Chamberlain RS, Sond J, Mahendraraj K, Lau CS, Siracuse BL, et al. (2018) Determining 30-day readmission risk for heart failure patients: the Readmission After Heart Failure scale. Int J Gen Med 11: 127-141.

7. King L HK, Dragan KL, Driver CR, Harris TG, Gwynn RC, et al. (2015) Community Health Profiles 2015. New York: Brooklyn Community District 17: East Flatbush

8. Verhaegh KJ, MacNeil-Vroomen JL, Eslami S, Geerlings SE, de Rooij SE, et al. (2014) Transitional care interventions prevent hospital readmissions for adults with chronic illnesses. Health Aff (Millwood) 33: 1531-1539.

9. Feltner C, Jones CD, Cene CW, Zheng ZJ, Sueta CA, et al. (2014) Transitional care interventions to prevent readmissions for persons with heart failure: a systematic review and meta-analysis. Ann Intern Med 160: 774-784.

10. Stamp KD, Machado MA, Allen NA (2014) Transitional care programs improve outcomes for heart failure patients: an integrative review. J Cardiovasc Nurs 29: 140-154.

11. Gorthi J, Hunter CB, Mooss AN, Alla VM, Hilleman DE, et al. (2014) Reducing Heart Failure Hospital Readmissions: A Systematic Review of Disease Management Programs. Cardiol Res 5: 126-138.

12. Oddone $E Z$, Weinberger $M$, Giobbie-Hurder $A$, Landsman $P$, Henderson $W$ et al. (1999) Enhanced access to primary care for patients with congestive heart failure. Veterans Affairs Cooperative Study Group on Primary Care and Hospital Readmission. Eff Clin Pract 2: 201-209.

13. Englander $H$, Michaels L, Chan B, Kansagara D (2014) The care transitions innovation (C-TraIn) for socioeconomically disadvantaged adults: results of a cluster randomized controlled trial. J Gen Intern Med 29: 1460-1467.

14. Ledwidge M, Barry M, Cahill J, Ryan E, Maurer B, et al. (2003) Is multidisciplinary care of heart failure cost-beneficial when combined with optimal medical care? Eur J Heart Fail 5: 381-389.

15. de la Porte PW, Lok DJ, van Veldhuisen DJ, van Wijngaarden J, Cornel JH, et al. (2007) Added value of a physician-and-nurse-directed heart failure clinic: results from the Deventer-Alkmaar heart failure study. Heart 93: 819-825.

16. Doughty RN, Wright SP, Pearl A, Walsh HJ, Muncaster S, et al. (2002) Randomized, controlled trial of integrated heart failure management: The Auckland Heart Failure Management Study. Eur Heart J 23: 139-146.
17. Capomolla S, Febo O, Ceresa M, Caporotondi A, Guazzotti G, et al. (2002) Cost/utility ratio in chronic heart failure: comparison between heart failure management program delivered by day-hospital and usual care. J Am Coll Cardiol 40: 1259-1266.

18. Stromberg A, Martensson J, Fridlund B, Levin LA, Karlsson JE, et al. (2003) Nurse-led heart failure clinics improve survival and self-care behaviour in patients with heart failure: results from a prospective, randomised trial. Eur Heart J 24: 1014-1023. 